\title{
Psychological and immunological characteristics of fatigued women undergoing radiotherapy for early-stage breast cancer
}

\author{
Nicholas Courtier • Tina Gambling • \\ Stephanie Enright • Peter Barrett-Lee • \\ Jacinta Abraham $\cdot$ Malcolm D. Mason
}

Received: 24 November 2011 / Accepted: 20 May 2012 / Published online: 28 May 2012

(C) Springer-Verlag 2012

\begin{abstract}
Purpose The amelioration of fatigue in radiotherapy patients is limited by an equivocal aetiology and uncertainty regarding who is likely to experience significant fatigue. The research objective was to characterise fatigue in women undergoing radiotherapy for breast cancer, in order to evaluate associations with elevations in anxiety, depression and a marker of systemic inflammation.

Methods Participants comprised 100 women, diagnosed with stages 0 -IIA breast cancer and prescribed with 40 Gy in 15 fractions over 3 weeks. Fatigue was assessed at baseline between 10 and 22 days before radiotherapy, after 10 and 15 fractions of radiotherapy and 4 weeks after the completion of radiotherapy, using the Functional Assessment of Chronic Illness Therapy Fatigue Subscale. Psychological status was self-reported using the Hospital Anxiety and Depression Scale. Sera concentrations of interleukin-6soluble receptor were established via enzyme-linked immunosorbent assay. The contributions of pretreatment factors to fatigue were analysed using multivariable regression.

Results Thirty-eight percent of participants experienced significant fatigue during radiotherapy, with the remainder little
\end{abstract}

\footnotetext{
N. Courtier $\cdot$ T. Gambling $\cdot$ S. Enright

School of Healthcare Studies, Cardiff University,

Cardiff, UK

P. Barrett-Lee · J. Abraham • M. D. Mason

Velindre Cancer Centre, Velindre NHS Trust,

Cardiff, UK

N. Courtier $(\square)$

Department of Radiography, School of Healthcare Studies,

Cardiff University,

Heath Park,

Cardiff CF14 4XN, UK

e-mail: CourtierN@cardiff.ac.uk
}

are affected. After controlling for baseline fatigue, anxiety before treatment was the strongest unique predictor of subsequent fatigue. During radiotherapy, interleukin-6-soluble receptor was significantly elevated in the fatigued group compared to the non-fatigued group $(p=0.01)$. This association was not mediated by depression.

Conclusions The data are consistent with the concept that psychological distress prior to radiotherapy relates to a distinct immunological and behavioural response during radiotherapy. Patients reporting elevated anxiety should benefit from interventions that appropriately address the underlying psychological distress and have the potential to ameliorate disabling treatment-related fatigue.

Keywords Fatigue · Breast cancer · Radiotherapy · Psychological mood $\cdot$ Inflammation

\section{Introduction}

Fatigue is the predominant determinant of health-related quality of life in women with breast cancer prescribed with curative radiotherapy [1]. Radiotherapy-related fatigue (RRF) adversely affects physical, cognitive and affective functioning, even years after the completion of treatment $[2,3]$, with profound repercussions for family structure, social life and finances. Despite patients' perception of fatigue as the most distressing symptom of radiotherapy [4], an equivocal aetiology renders the symptom largely untreated.

Depression is a known correlate of fatigue in patients with breast cancer [5-7]. Whether depressed mood antecedes cancer-related fatigue or vice versa or the symptoms coexist as components of a treatment-related symptom cluster remains ambiguous. 
Some degree of conceptual overlap is evident between the International Classification of Diseases-10 criteria for cancer-related fatigue (CRF)/National Comprehensive Cancer Network CRF Guidelines and diagnostic criteria for depressive disorder [8-10]. However, distinguishing aspects are evident. Depression is less prevalent than CRF in cancer patients [11]. Whilst the presence of fatigue and psychological distress are largely attributable to events surrounding diagnosis and treatment [11], a number of studies have suggested that fatigue and depressed mood either exhibit divergent chronologies and trajectories [12-14] or have independent determinants $[15,16]$. A distinctive feature of CRF symptomology is a generalised sensation of weakness, which is associated with an extra-ordinary amount of effort required to complete motor tasks [16]. Conversely, anhedonia is a common feature of depressive disorder but not CRF [10].

Basic evidence for the concept that depression and fatigue coexist due to a common causative factor [17-19] is provided by reports that the therapeutic administration of cytokines renders symptom clusters involving fatigue and depression [20, 21]. The cytokine interleukin-6 (IL-6) is the principal mediator of this inflammatory process. Very limited cells express receptors for IL-6. However, when IL-6 dimerises with its agonistic soluble receptor (interleukin-6soluble receptor (IL-6sR)), ubiquitous cell signalling from the periphery to the central nervous system is enabled; via a process called trans-signalling [22]. Trans-signalling provides a theoretical basis for central effects from localised irradiation.

The objective of this research was to examine the characteristics of fatigued women undergoing radiotherapy for breast cancer in order to evaluate associations with elevated psychological distress and systemic IL-6sR. Translational implications for fatigue attenuation will be discussed.

\section{Methods and materials}

\section{Study design}

A cohort study with observations was made 10-22 days before radiotherapy, the end of weeks 2 and 3 of radiotherapy and 4 weeks post-radiotherapy.

\section{Participants}

The study population comprised women diagnosed with histologically confirmed early-stage carcinoma of the breast, having undergone primary surgery and subsequently referred to Velindre Cancer Centre for adjuvant radiotherapy. All participants were prescribed with 4,000 cGy in 15 fractions over 3 weeks. Exclusion criteria included thyroid dysfunction; inflammatory diseases; uncontrolled heart; lung or liver disease; a history of significant or untreated depression; prior or concurrent systemic endocrine or cytotoxic therapy; and advanced disease. The South East Wales Research Ethics Committee approved the study.

\section{Measures}

Socio-demographic, disease and treatment-related variables were obtained from hospital records. Fatigue intensity over the previous 7 days was measured using the Functional Assessment of Chronic Illness Therapy Fatigue Scale (FACIT-F). The format of the scale is a 13-item questionnaire using a Likert-type scale. The items are summed to generate a cumulative score between 0 (maximum fatigue) and 52 (no fatigue) [23]. A minimally clinically significant change is defined as three to four points [24].

When the average of scores at weeks 2 and 3 of radiotherapy was $\leq 34$, participants were categorised as fatigued and $35+$ as non-fatigued. The cut-off score of 34 was adopted as the most conservative value proposed previously $[25,26]$. Such summary measures tend to counteract classification bias, response shift and recency, and overcomes the statistical problem of non-independent observations over time.

The Hospital Anxiety and Depression Scale (HADS) provided a self-reported measure of anxiety and depression over the previous 7 days. This widely validated tool poses 14 questions alternately pertaining to anxiety or depression. Responses to the questions are scored between 0 (none) to 3 (severe), generating a resultant score between 0 and 21 for each symptom. Symptom scores can be categorised as 'normal' (0-7), 'borderline' (8-10) and 'probable case' (11-21) [27].

The researcher scheduled brief meetings with the participants on the days when questionnaires were due to prompt their return. Whilst participants were informed in broad terms, they were unaware of specific research questions. Conversely, the researcher remained blind to the fatigue status of the participant until all other measurements were complete. These strategies acted as safeguards against bias that can weaken observational study designs.

Aliquots of serum were collected in accordance with standard protocols and immediately stored at $-70^{\circ} \mathrm{C}$ until analysed. Commercially available enzyme-linked immunosorbent assay (ELISA) kits, optimised for use with human serum, determined circulating concentrations of IL-6sR (R\&D Systems Quantikine ELISA Kits, Minneapolis, USA).

The rationale for assaying IL-6sR was fourfold: (a) Primary acute phase effects are reflected in IL- 6 activity [22, 28]; (b) Elevated IL-6 is associated with CRF, as well as anxiety and depression [29]; (c) In contexts where IL-6 has 
adverse (chronic or excessive action) pro-inflammatory effects, the action of IL-6 is mediated by IL-6sR [30], via trans-signalling. The central effects of IL- 6 depend largely on peripheral concentrations of IL-6sR; and (d) Positive associations have been demonstrated between IL-6sR and fatigue in survivors of breast cancer [19, 31].

Sample size and statistical methods

A projected recruitment rate of two per week and a period of 12 months dictated a pragmatic sample size of 100 . A power calculation, based on a multiple regression test with five predictors, a moderate effect size $f^{2}=0.15$ and significance level $\alpha=0.05$ and a sample size of 100 would render power of $84 \%$ [32].

Analyses were conducted using SPSS version 16. All tests were $n=100$ unless otherwise stated. Group comparisons were made using the Mann-Whitney test or one-way between-groups ANOVA, and longitudinal comparisons were conducted using the Friedman test. Bivariate associations were assessed by Spearman's rank order correlations. Baseline variables were selected for inclusion entry into a multiple regression model by a forced-entry method. Model residuals and scatterplots were examined to check if the data fulfilled the test's underlying assumptions.

\section{Results}

Between November 2007 and October 2008, 130 eligible women were consecutively approached to participate in the study. Fifteen women were precluded from participation on the basis of a full medical history; most commonly a history of rheumatoid arthritis. A further 15 eligible women declined to participate. Eight did not wish to undergo blood sampling, four declared a resistance to thinking about 'extra things', with the remaining three declining to volunteer a reason. The mean (standard deviation (SD)) age and median (inter-quartile range (IQR)) pretreatment FACIT-F score recorded for 12 eligible non-entrants were 57.1 (7.4) and 43 (37-46), respectively. All other women gave their informed consent prior to their inclusion in the study.

Fatigue group characteristics

The median FACIT-F fatigue scores for the whole cohort decreased from 44 at baseline to 41 at week 2, reaching a nadir of 37 at week 3 , with a subsequent improvement to 42 at 4-week follow-up. When the cohort was divided based on the criteria for a fatigue case, $38 \%$ of participants were categorised as fatigued, with the remaining $62 \%$ classified as non-fatigued. The median FACIT-F response for the two groups is shown in Fig. 1. The grouped participant characteristics are presented below in Table 1 .

Figure 1 obscures the fact that $27 \%$ of subjects recorded decreased fatigue at week 3 as compared to baseline. The majority (22) of these 'improvers', were in the non-fatigued group, exhibiting a modest improvement of up to five-scale points. Conversely, $26 \%$ of participants remained categorised as fatigued at follow-up.

\section{Psychological mood}

HADS depression data classified $92 \%$ as normal, $4 \%$ as borderline and only a further $4 \%$ at the lower bounds of the probable case range. By week 3, these proportions were 79 , 14 and $7 \%$, respectively. The corresponding figures for anxiety were 75,12 and $13 \%$ at baseline and 73,14 and $13 \%$ at week 3. Median (IQR) HADS scores for the whole cohort were relatively stable from baseline to week 3 , being $5(3-7)$ to $4(2-7)$ and $2(1-5)$ to $3(1-6)$ for anxiety and depression, respectively. The relationships between fatigue group and psychological mood are illustrated in Figs. 2 and 3.

Although the reduction in anxiety over time was parallel for the two groups, results of the Friedman test indicated a
Fig. 1 Median longitudinal FACIT-F scores for fatigued and non-fatigued groups. (Error bars $=$ inter-quartile range; percentages $=$ the proportion of the cohort classified in each group)

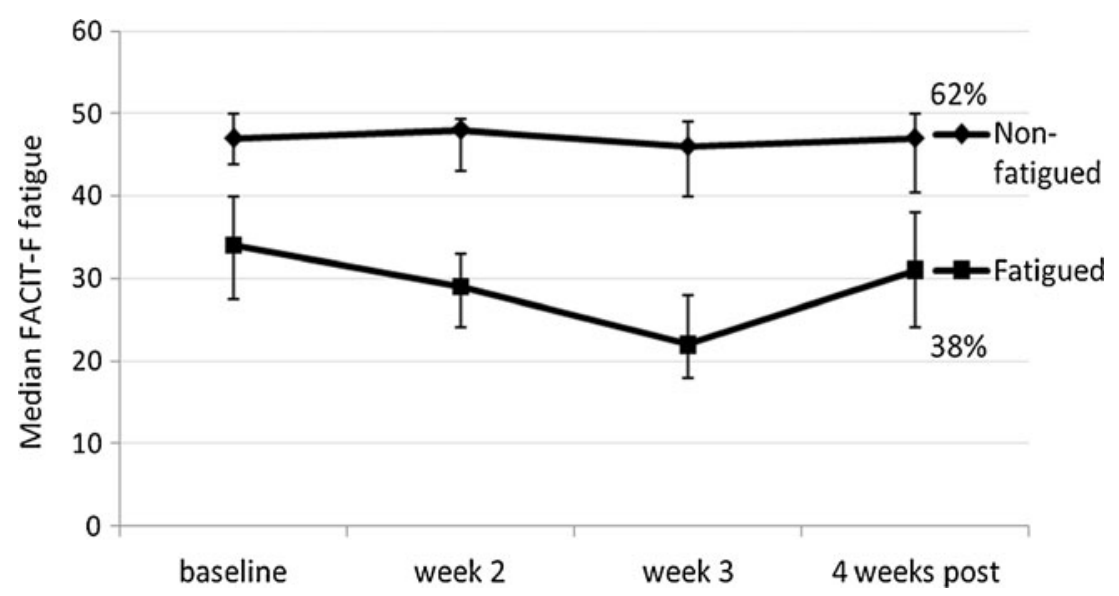


Table 1 Pre-radiotherapy sample characteristics for fatigued and nonfatigued groups

\begin{tabular}{|c|c|c|}
\hline Characteristic & $\begin{array}{l}\text { Fatigued group } \\
(n=38)\end{array}$ & $\begin{array}{l}\text { Non-fatigued } \\
\text { group }(n=62)\end{array}$ \\
\hline Age (years) & $55.9(9.3)$ & $59.0(8.5)$ \\
\hline BMI $\left(\mathrm{Kg} / \mathrm{m}^{2}\right)$ & $29.2(4.8)$ & $27.6(4.6)$ \\
\hline \multicolumn{3}{|l|}{ Work status } \\
\hline Retired/housewife & $24(63.2 \%)$ & $34(54.8 \%)$ \\
\hline Continued to work during RT & $2(5.3 \%)$ & $6(9.7 \%)$ \\
\hline Postponed work during RT & $12(31.6 \%)$ & $22(35.5 \%)$ \\
\hline \multicolumn{3}{|l|}{ Menopausal status } \\
\hline Post-menopausal & $25(65 \%)$ & $48(77.4 \%)$ \\
\hline Peri-menopausal & $7(18.4 \%)$ & $9(14.5 \%)$ \\
\hline Pre-menopausal & $6(15.8 \%)$ & $5(8.1 \%)$ \\
\hline \multicolumn{3}{|l|}{ HRT history } \\
\hline Never & $25(65.8 \%)$ & $45(72.6 \%)$ \\
\hline Previous & $13(24.2 \%)$ & $17(27.4 \%)$ \\
\hline \multicolumn{3}{|l|}{ Smoking history } \\
\hline Never & $16(42.1 \%)$ & $34(54.8 \%)$ \\
\hline Past & $14(36.8 \%)$ & $18(29 \%)$ \\
\hline Current & $8(21.1 \%)$ & $10(16.1 \%)$ \\
\hline Pack-years ${ }^{\mathrm{a}}$ & $12.6(16.4)$ & $6.2(9.7)$ \\
\hline \multicolumn{3}{|l|}{ Histological diagnosis } \\
\hline Ductal carcinoma in situ & $6(15.8 \%)$ & $7(11.3 \%)$ \\
\hline Invasive ductal carcinoma & $18(47.4 \%)$ & $43(69.4 \%)$ \\
\hline Invasive lobular carcinoma & $5(13.2 \%)$ & $7(11.3 \%)$ \\
\hline Other & $9(23.7 \%)$ & $5(8.1 \%)$ \\
\hline Tumour size $(\mathrm{mm})^{\mathrm{a}}$ & $17(10.1)$ & $18.4(10.4)$ \\
\hline \multicolumn{3}{|l|}{ Histopathological grade } \\
\hline Grade 1 & $14(36.8 \%)$ & $17(27.4 \%)$ \\
\hline Grade 2 & $17(44.7 \%)$ & $34(54.8 \%)$ \\
\hline Grade 3 & $7(18.4 \%)$ & $11(17.7 \%)$ \\
\hline \multicolumn{3}{|l|}{ TNM stage } \\
\hline 0 & $7(18.4 \%)$ & $6(9.7 \%)$ \\
\hline I & $25(65.8 \%)$ & $45(72.6 \%)$ \\
\hline IIA & $6(15.8 \%)$ & $11(17.7 \%)$ \\
\hline \multicolumn{3}{|l|}{ Laterality } \\
\hline Right & $20(52.6 \%)$ & $32(51.6 \%)$ \\
\hline Left & $18(47.4 \%)$ & $30(48.4 \%)$ \\
\hline Time from surgery to RT (days) & $61.5(12.2)$ & $61.0(16.7)$ \\
\hline
\end{tabular}

Normally distributed continuous variables are means (standard deviation). Categorical variables are numbers (and percentages) of patients in that group

$B M I$ body mass index, HRT hormone replacement therapy, $R T$ radiotherapy

${ }^{a}$ Non-normally distributed continuous variables are medians (interquartile range)

statistically significant reduction in anxiety score for the larger non-fatigued group, $\chi^{2}(2, n=62)=6.9, p=0.03$, in contrast to $\chi^{2}(2, n=38)=0.5, p=0.8$ for the fatigued group.
The results of the Friedman test indicated a statistically significant increase in depression score for the fatigued group, $\chi^{2}(2, n=38)=19, p<0.005$, but not for the non-fatigued group $\chi^{2}(2, n=62)=1.3, p=0.8$.

\section{IL-6sR concentrations}

Heterogeneity was evident in both the baseline sera IL-6sR concentrations and longitudinal changes from baseline. IL6sR concentration increased for $68 \%$ of participants and decreased for the remaining $32 \%$ during radiotherapy. Median data are presented for the two fatigue groups in Fig. 4. (Note that the error bars represent inter-quartile range and are therefore not indicative of statistical significance.)

Mann-Whitney $U$ tests revealed statistically significant differences in the group IL-6sR concentrations at weeks 2 $(U=854, z=-2.30, p=0.02)$ and $3(U=837, z=-2.42, p=$ 0.01 ), but not pre- or post-treatment. Associated effect sizes were moderate, $r \approx 0.3$. As 12 women declined to return for a blood test after treatment, the 4-week follow-up IL-6sR data were $n=88$.

Inter-relationships between variables

As a prelude to multivariable analysis, bivariate (Spearman's rank correlation) analyses were conducted for all time points. The strongest statistical correlation with baseline FACIT-F score was a negative correlation with depression score, $\rho=-0.71, p<0.001$. The relationship between anxiety and fatigue was approximately half the strength, $\rho=-0.35$, $p<0.001$. Older age was significantly associated with lower anxiety, $\rho=-0.27, p=0.004$, but not with depression level, $\rho=-0.009, p=0.9$. Statistically significant correlations were also revealed between baseline IL-6sR concentration and both fatigue, $\rho=-0.30, p=0.003$, and depression, $\rho=0.29$, $p=0.003$.

To determine if the correlation between baseline fatigue and IL-6sR was influenced by associations between the latter and depression, an exploratory Pearson's partial correlation analyses were undertaken. Histograms and scatter plots suggested assumptions of normality, linearity and homoscedasticity were reasonably met. The zero-order correlation, $r=-0.32, p=0.002$, was rendered statistically nonsignificant, $r=-0.07, p=0.5$, when controlling for depression. In contrast, the zero-order correlation between IL-6sR and fatigue at week $3, r=-0.31, p=0.005$, was unchanged when controlling for depression level.

The apparent influence of depression on the relationship between baseline fatigue and IL-6sR concentration instigated exploratory one-way between-groups ANOVA. Low, medium and high depression groups were formed by dividing the cohort into ordinal thirds. A statistically significant difference was evident in IL-6sR concentration for the three 
Fig. 2 Median longitudinal HADS anxiety scores for fatigued and non-fatigued groups. (Error bars $=$ interquartile range)

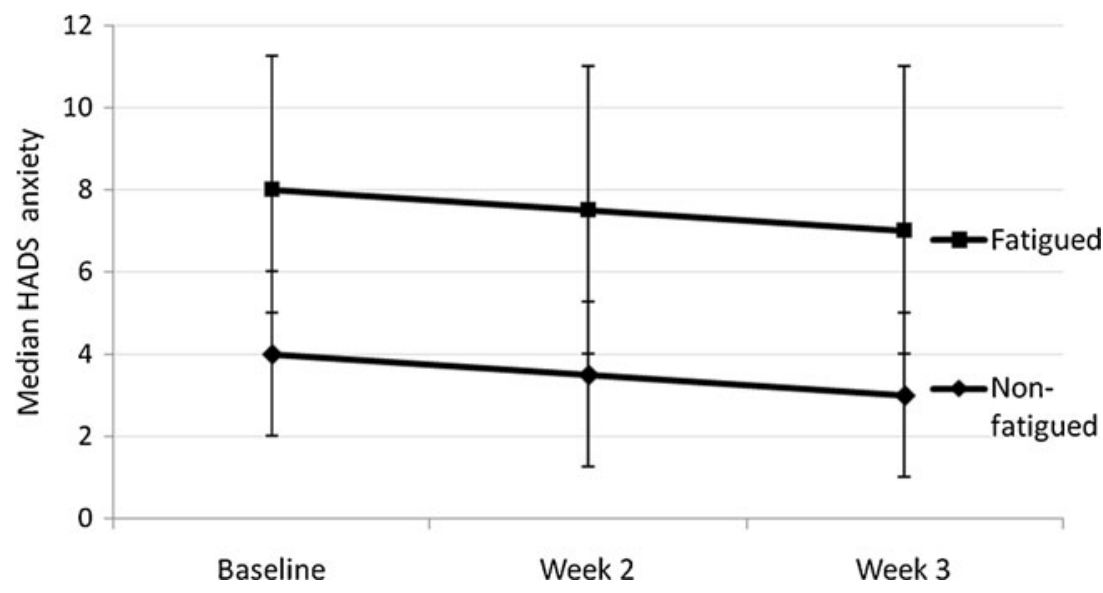

groups: $F(2,97)=4.5, p=0.01, \eta^{2}=0.9$ (moderate effect size). Tukey's HSD post hoc comparisons indicated that the mean (SD) concentration for the high depression group, $M=46.3 \mathrm{ng} / \mathrm{mL}$ (11.6), was significantly different from the low group, $M=37.9 \mathrm{ng} / \mathrm{mL}$ (11.5), but the moderate group, $M=41.2 \mathrm{ng} / \mathrm{mL}$ (9.2), did not differ significantly from the other two. An equivalent analysis was conducted for baseline anxiety: no significant difference was found between any groups: $F(2,97)=2.1, p=0.1$.

A standard multiple regression analysis determined the relative contribution of baseline risk factors to fatigue during radiotherapy. The dependent variable was the mean of the fatigue scores at weeks 2 and 3. Two models were computed: model 1 with baseline fatigue and model 2 without baseline fatigue. The results are summarised in Table 2 .

\section{Discussion}

Fatigue

The cohort reported a modest, but clinically significant, increase of seven points in median fatigue at the end of radiotherapy compared to baseline. Based on the adopted fatigue cut-off, $38 \%$ of participants were categorised as fatigued, with the remainder categorised as non-fatigued. Categorisation demonstrates that the non-fatigued group remains virtually unaffected from baseline levels of fatigue, which are comparable to normative population data for agematched females [33].

Participants who were not fatigued before treatment tend to remain so. In comparison, the fatigued subgroup is significantly more fatigued before radiotherapy and fatigue intensifies throughout treatment. The group difference in median scores was 13 at baseline, 23 at week 3, closing to 16 four weeks later. The trend for recovery after treatment is reassuring. However, considerable variance in post-treatment trajectory was evident, with $26 \%$ still categorised as fatigued at 4 weeks follow-up and ten subjects reporting a clinically significant deterioration of four points or more.

These fatigue response data are important as these reflect the current NICE-recommended UK breast radiotherapy fractionation schedule. Whilst fatigue intensity may be attenuated compared to longer international schedules [12, 26], both the prevalence and the course of fatigue are broadly in accordance with the closest comparator [26], which reported that $43 \%$ experience significant fatigue. This slightly higher proportion was derived using a less stringent
Fig. 3 Median longitudinal HADS depression scores for fatigued and non-fatigued groups. (Error bars $=$ interquartile range)

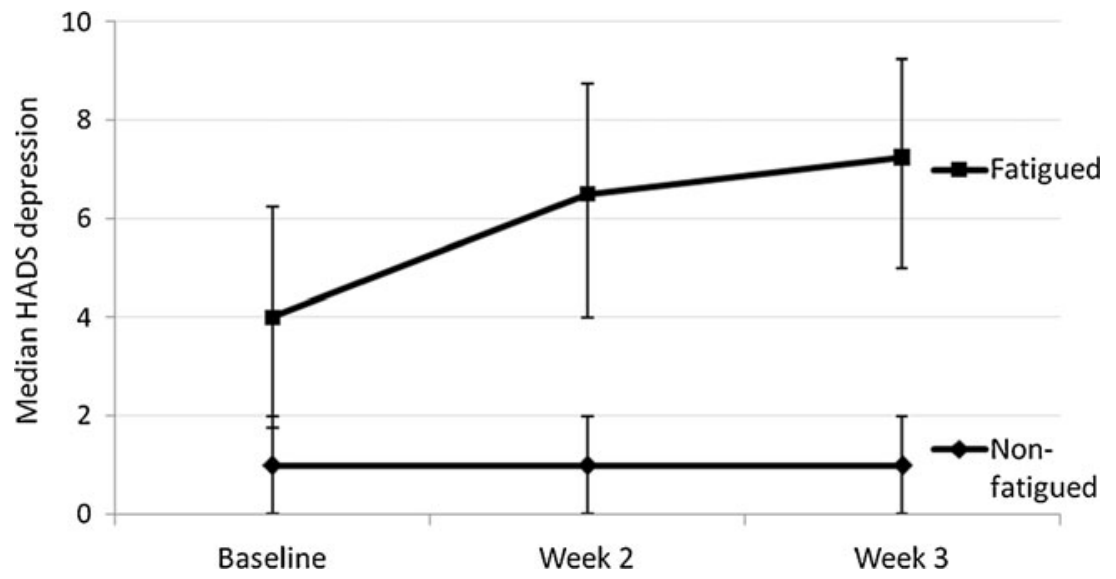


Fig. 4 Median longitudinal sera IL-6sR concentrations for fatigued and non-fatigued groups. (Error bars $=$ interquartile range; $I L-6 s R=$ interleukin-6 soluble receptor

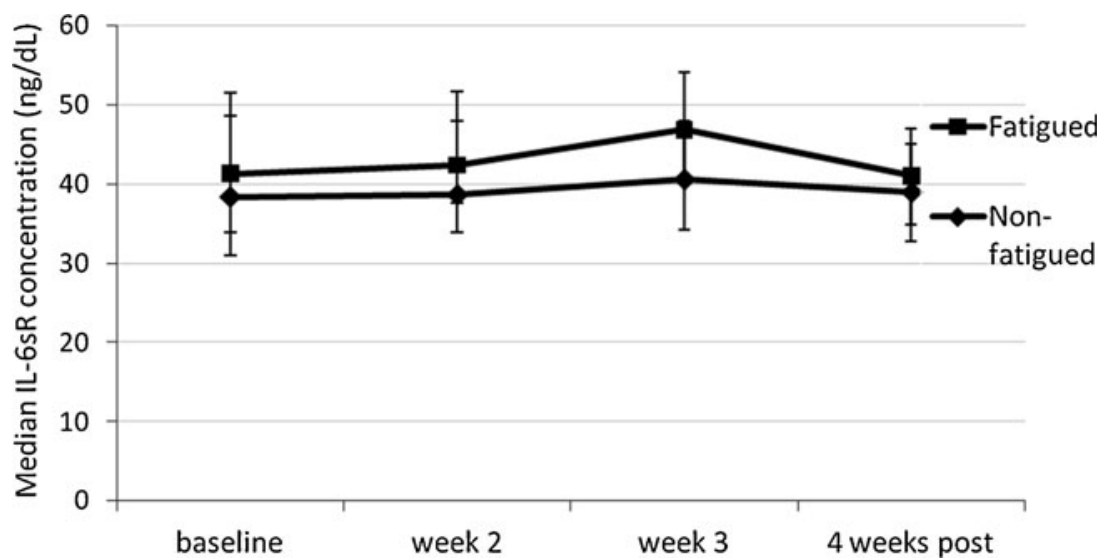

Anxiety scores for the fatigued group were higher at baseline than for the non-fatigued group - median scores being 8 and 4, respectively. By week 3 of treatment, both groups had recorded a decrease of one point-presumably due to familiarisation with the realities of treatment. The parallel longitudinal course for the two fatigue groups indicates that anxiety primarily affects baseline fatigue level, and the increase in subsequent fatigue reported by the fatigued group is not attributable to changes in anxiety during treatment.

Baseline depression score was the factor that contributed most to fatigue level before radiotherapy, after controlling for other factors. When considering the contribution of baseline variables to on-treatment fatigue, the inclusion of baseline fatigue increases the proportion of fatigue explained from 37 to $47 \%$. Considerable overlap is also evident in the variance explained by fatigue and depression. The proportion of ontreatment fatigue that depression uniquely explains decreases from $12 \%$ to a negligible $0.2 \%$. Anxiety actually makes the largest unique contribution.

It is worthwhile cautioning that multiple regression is based on correlation and no definitive causative claims should be made. However, hypothesis generation can be supported by a consideration of the strength and temporality of relationships and underlying mechanisms. Fatigue is ultimately a centrally driven phenomenon. Research at the interface of psychoneuroimmunology has coalesced around the concept that stressors modulate bidirectional communication between a peripheral inflammatory event (e.g. radiotherapy) and the central nervous system [35-37].

Psychological stress may plausibly precipitate/potentiate RRF via two mechanisms. First, a review of the biological effects of psychological interventions in breast cancer patients identified psychological stress-related dysregulation of the neuroendocrine system (cortisol) as the principal mediator of relevant biological processes [38]. It has been demonstrated that fatigued breast cancer survivors have a suppressed cortisol response to acute psychological stress [39, 40]. As cortisol is a potent hormonal suppressor of the inflammatory response, it is not surprising that suppressed cortisol activity is associated 
with augmented IL-6 activity in patients with breast cancer [41]. A secondary pathway that may enable anxiety to gain control of fatigue responses is via poor health behaviours associated with stress, such as increased smoking and reduced exercise [38, 42].

\section{IL-6sR}

A normal serum range quoted for healthy populations is 15.2 to $45.0 \mathrm{ng} / \mathrm{mL}$, with a mean of $25.5 \mathrm{ng} / \mathrm{mL}$ [43]. No comparative results for IL-6sR concentration during radiotherapy could be identified. Data for breast cancer survivors between 1 and 5 years post-radiotherapy are available [31]: mean plasma concentrations of 30.6 and $40.1 \mathrm{ng} / \mathrm{mL}$ were reported for non-fatigued and fatigued participants, respectively. The current study recorded mean sera concentrations that were comparable to the latter at baseline and approximately $20 \%$ higher at week 3 .

Variance in IL-6sR samples made it difficult to discern if a partial elevation during radiotherapy was a substantive finding. Certainly, statistically significant differences between fatigue groups were only evident during treatment. Further analyses suggested that IL-6sR concentration was more directly associated with fatigue during radiotherapy, in contrast to a relationship mediated by depression before radiotherapy. IL-6sR was related to depression score, but not anxiety, in the absence of irradiation.

Overall, the study data are consistent with theory suggesting that psychological distress can sensitise the immunological and behavioural response to subsequent inflammatory events [7, 37, 38, 42]. Women in this study with high levels of perceived stress before radiotherapy - in response to diagnosis, surgery, life factors, etc.- - were most at risk for neurobehavioural problems such as fatigue.

\section{Study limitations}

A strength of this study is the homogeneity of the sample. However, the study findings should strictly only be generalised to comparable patient groups. Whilst relevant comorbidities were excluded, no attempt was made to control the use of medication that may be a confounder of inflammation and/or fatigue.

This study cannot determine whether the participants whose fatigue levels did not improve post-treatment continued on a downward trajectory. Evidence does suggest that fatigue levels during the acute phase are a strong risk factor for experiencing chronic fatigue $[2,4]$.

To comprehensively profile the inflammatory response to radiotherapy would require a range of biomarkers to be assayed. The degree to which peripheral cytokine concentrations reflect local concentrations is also at issue. Furthermore, cytokine release is known to be affected by circadian, diurnal and menstrual rhythms, none of which were controlled for. The time of day and fasting effects appear to have no material effect on short-term variability of IL-6sR [43].

Implications for theory and practice

The divergent course of fatigue groups suggests that baseline factors are determinants of fatigue that radiotherapy consequentially exacerbates. Screening of predictors of fatigue before treatment would enable the majority of patients to be reassured that fatigue is likely to be mild and transitory. For a significant minority of women, we hypothesise that the anxiety response affects fatigue before treatment and sensitises for subsequent behavioural symptoms. Levels of anxiety and fatigue that indicate a need for enhanced support are thus sustained throughout treatment.

The current study has not yet provided definitive evidence of a mediating role of inflammatory processes. However, a psychological distress response antecedes neuroimmunological changes, plausibly via cortisol modulation. Whatever the mechanisms, the implication is that interventions to address fatigue should focus on distress reduction, which should be instigated prior to radiotherapy. Efficacy may be promoted by providing coping techniques rather than fundamental change of relatively stable personality traits [7]. The active components of psychological interventions have been previously elucidated [44], although much uncertainty remains regarding the optimal delivery format.

Even the most active intervention will show insufficient promise when tested in the majority of women who report negligible fatigue (and anxiety and depression) both before and during radiotherapy. Instead, help should be targeted at women at a high risk of experiencing fatigue. This study, and others [34], indicates that younger women may find the prospect of daily confrontation with their disease particularly distressing. As age acts as a proxy for menopausal status, it may be relevant that a larger proportion of pre-/peri-menopausal women were in the fatigued group relative to the non-fatigued group. Deprivation and social support are additional contextual factors likely to modulate anxiety responses [45].

Further research should extend into the chronic period to discern if acute changes in fatigue are sustained. The measurement of waking cortisol concentrations in future work could provide useful mechanistic evidence of intervening variables. Structural equation modelling would be an appropriate method to test a proposed 'causative' model. Targeting of evidence-based treatment of fatigue is vital to maximise the quality of life after curative radiotherapy.

Acknowledgments The study was funded by the Research Capacity Building Collaboration Wales and the College of Radiographers Industrial Partnership Scheme Research Awards. We would like to thank the Cardiff University staff at the Cancer Research Wales laboratories for assistance with the cytokine assays. 
Disclosures None.

\section{References}

1. Dagnelie PC, Pijls-Johannesma MCG, Lambin P, Beijar S, De Ruysscher D, Kempen GIJM (2007) Impact of fatigue on overall quality of life in lung and breast cancer patients selected for highdose radiotherapy. Ann Oncol 18:940-944

2. Geinitz H, Zimmermann FB, Thamm R, Keller M, Busch R, Molls M (2004) Fatigue in patients with adjuvant radiation therapy for breast cancer: long-term follow-up. J Cancer Res Oncol 130:327-333

3. Bower JE, Ganz PA, Desmond KA, Bernaards C, Rowland JH, Meyerowitz BE, Belin TR (2006) Fatigue in long-term breast carcinoma survivors. Cancer 106:751-758

4. Curt GA, Breitbart W, Cella D, Groopman JE, Horning SJ, Itri LM, Johnson DH, Miaskowski C, Scherr SL, Portenoy RK, Vogelzang NJ (2000) Impact of cancer-related fatigue on the lives of patients: new findings from the fatigue coalition. Oncologist 5:353-360

5. Bennett B, Goldstein D, Lloyd A, Davenport T, Hickie I (2004) Fatigue and psychological distress - exploring the relationship in women treated for breast cancer. Eur J Cancer 40:1689-1695

6. De Vries J, Van der Steeg AF, Roukema JA (2009) Trait anxiety determines depressive symptoms and fatigue in women with an abnormality in the breast. Brit J Health Psychol 14:143-157

7. Jacobsen PB, Donovan KA, Weitzner MA (2003) Distinguishing fatigue and depression in patients with cancer. Sem Clin Neuropsychiatry 8:229-240

8. Portenoy RK, Itri LM (1999) Cancer-related fatigue: guidelines for evaluation and management. Oncologist 4:1-10

9. National Comprehensive Cancer Network (2010) Cancer-related fatigue. NCCN Clinical Practice Guidelines in Oncology, version 1. Available online: http://www.nccn.org/professionals/physician_gls/ f_guidelines.asp\#site

10. American Psychiatric Association (1994) Diagnostic and statistical manual of mental disorders, 4th edn. American Psychiatric Press, Washington

11. Goldstein D, Bennett B, Friedlander M, Davenport T, Hickie I, Lloyd A (2006) Fatigue states after cancer treatment occur both in association with, and independent of, mood disorder: a longitudinal study. BMC Cancer 1:240-248

12. Geinitz H, Zimmermann FB, Stoll P, Thamm R, Kaffenberger W, Ansorg K, Keller M, Busch R, Beruningen DK, Molls M (2001) Fatigue, serum cytokine levels, and blood cell counts during radiotherapy of patients with breast cancer. Int J Rad Oncol Biol Phys 51:691-698

13. Knobf MT, Sun Y (2005) A longitudinal study of symptoms and self-care activities in women treated with primary radiotherapy for breast cancer. Cancer Nurs 28:210-218

14. Visser MRM, Smets EMA (1998) Fatigue, depression and quality of life in cancer patients: how are they related? Support Care Cancer 6:101-108

15. Morrow GR, Hickok JT, Roscoe JA, Raubertas RF, Andrews PL, Flynn PJ, Hynes HE, Banerjee TK, Kirshner JJ, King DK (2003) Differential effects of paroxetine on fatigue and depression: a randomized, double-blind trial from the University of Rochester Cancer Center Community Clinical Oncology Program. J Clin Oncol 21:4635-4641

16. Cella DF, Peterman A, Passik S, Jacobsen PB, Breitart W (1998) Progress toward guidelines for the management of fatigue. Oncology 12:369-377

17. Kim HJ, Barsevick AM, Tulman L, McDemott PA (2008) Treatment-related symptom clusters in breast cancer: a secondary analysis. J Pain Symptom Manage 36:468-479
18. Cleeland CS, Bennett GJ, Dantzer R, Dougherty PM, Dunn AJ, Meyers CA, Miller AH, Payne R, Reuben JM, Wang XS, Lee BN (2003) Are the symptoms of cancer and cancer treatment due to a shared biologic mechanism? A cytokine-immunologic model of cancer symptoms. Cancer 97:2919-2925

19. Bower JE (2008) Behavioural symptoms in patients with breast cancer and survivors. J Clinl Oncol 26:768-777

20. Capuron L, Gumnick JF, Musselman DL, Lawson DH, Reemsnyder A, Nemeroff CB, Miller AH (2002) Neurobehavioral effects of interferon-alpha in cancer patients: phenomenology and paroxetine responsiveness of symptom dimensions. Neuropsychopharmacology 26:643-652

21. Capuron L, Ravaud A, Miller AH, Dantzer R (2004) Baseline mood and psychosocial characteristics of patients developing depressive symptoms during interleukin-2 and/or interferon-alpha cancer therapy. Brain Behav Immun 18:205-213

22. Scheller J, Ohnesorge N, Rose-John S (2006) Interleukin-6 transsignalling in chronic inflammation and cancer. Scan J Immunol 63:321-329

23. Cella D (1997) Manual of the Functional Assessment of Chronic Illness Therapy (FACIT) measurement system. Evanston Northwestern Healthcare and Northwestern University, Evanston

24. Cella DF, Eton DT, Lai SJ, Peterman A, Merkel DE (2002) Combining anchor and distribution-based methods to derive minimal clinically important differences on the Functional Assessment of Cancer Therapy (FACT) anemia and fatigue scales. J Pain Symptom Manage 24:547-561

25. Van Belle S, Paridaens R, Evers G, Kerger J, Bron D, Foubert J, Ponnet G, Steichel DV, Heremans C, Rosillon D (2005) Comparison of proposed diagnostic criteria with FACT-F and VAS for cancer related fatigue: proposal for use as a screening tool. Support Care Cancer 13:246-254

26. Wratten C, Kilmurray J, Nash S, Seldon M, Hamilton CS, O’Brien PC, Denham JW (2004) Fatigue during breast radiotherapy and its relationship to biological factors. Int J Rad Oncol Biol Phys 59:160-167

27. Zigmond AS, Snaith RP (1983) The hospital anxiety and depression scale. Acta Psychiatr Scan 67:361-370

28. Jones SA, Richards PJ, Scheller J, Rose-John S (2005) Review: IL6 transignalling: the in vivo consequences. J Interferon Cytokine Res 25:241-253

29. Schubert C, Hong S, Natarajan L, Mills P, Dimsdale JE (2007) The association between fatigue and inflammatory marker levels in cancer patients: a quantitative review. Brain Behav Immun 2:413-427

30. Jones S, Horiuchi S, Topley N, Yamamoto N, Fuller GM (2001) The soluble interleukin 6 receptor: mechanisms of production and implications in disease. FASEB 15:45-58

31. Collado-Hidalgo A, Bower JE, Ganz PA, Cole SW, Irwin MR (2006) Inflammatory biomarkers for persistent fatigue in breast cancer survivors. Clin Cancer Res 12:2759-2766

32. Faul F, Erdfelder E, Lang A-G, Buchner A (2007) G*Power 3: a flexible statistical power analysis program for the social, behavioral, and biomedical sciences. Behav Res Meth 39:175-191

33. Cella D, Lai S, Chang CH, Peterman A, Slavin M (2002) Fatigue in cancer patients compared with fatigue in the general United States population. Cancer 94:528-538

34. Hopwood P, Haviland J, Mills J, Sumo G, Bliss J (2007) The impact of age and clinical factors on quality of life in early breast cancer: an analysis of 2208 women recruited to the UK START Trial (Standardisation of Breast Radiotherapy Trial). Breast 16:241-251

35. Dantzer R, Kelley KW (2007) Twenty years of research on cytokineinduced sickness behaviour. Brain Behav Immun 21:153-160

36. Watkins LR, Maier SF, Goehler LE (1995) Cytokine-to-brain communication: a review and analysis of alternative mechanisms. Life Sci 57:1011-1026 
37. Miller AH, Ancoli-Israel S, Bower JE, Capuron L, Irwin MR (2008) Neuroendocrine-immune mechanisms of behavioural comorbidities in patients with cancer. J Clin Oncol 26:971-982

38. McGregor BA, Antoni MH (2009) Psychological intervention and health outcomes among women treated for breast cancer: a review of stress pathways and biological mediators. Brain Behav Immun 23:159-166

39. Bower JE, Ganz PA, Aziz N (2005) Altered cortisol response to psychologic stress in breast cancer survivors. Psychosom Med 67:277-280

40. Bower JE, Ganz PA, Dickerson SS, Petersen L, Aziz N, Fahey JL (2005) Diurnal cortisol rhythm and fatigue in breast cancer survivors. Psychoneuroendocrinology 30:92-100

41. Bower JE, Ganz PA, Aziz N, Olmstead R, Irwin MR, Cole SW (2007) Inflammatory responses to psychological stress in fatigued breast cancer survivors: relationship to glucocorticoids. Brain Behav Immun 21:251-258

42. Seruga B, Zhang H, Bernstein L, Tannock IF (2008) Cytokines and their relationship to the symptoms and outcome of cancer. Nat Rev Cancer 8:887-899

43. Dugué B, Leppänen E (1998) Short-term variability in the concentration of serum interleukin-6 and its soluble receptor in subjectively healthy persons. Clin Chem Lab Med 36:323-325

44. Goedendorp MM, Gielissen MF, Verhagen CA, Bleijenberg G (2009) Psychosocial interventions for reducing fatigue during cancer treatment in adults. Cochrane Database Syst Rev 21: CD006953

45. Macleod U, Ross S, Fallowfield L, Watt GCM (2004) Anxiety and support in breast cancer: is this different for affluent and deprived women? A questionnaire study. Brit J Cancer 91:879-883 